\title{
Comparative investigation on nanomechanical properties of hardened cement paste
}

\author{
Wengui Li $\cdot$ Shiho Kawashima $\cdot$ \\ Jianzhuang Xiao • David J. Corr • Caijun Shi • \\ Surendra P. Shah
}

Received: 30 April 2014/Accepted: 14 March 2015/Published online: 22 March 2015

(C) RILEM 2015

\begin{abstract}
Three types of nanomechanical methods including static nanoindentation, modulus mapping and peak-force quantitative nanomechanical mapping (QNM) were applied to investigate the quantitative nanomechanical properties of the same indent location in hardened cement paste. Compared to the nanoindentation, modulus mapping and peak-force QNM allow for evaluating local mechanical properties of a smaller area with higher resolution. Beside, the ranges of elastic modulus distribution measured by modulus mapping and peak-force QNM are relatively greater than that obtained from nanoindentation, which may be due to a result of the shaper probe and local confinement effect between multiple phases. Moreover, the average value of elastic modulus obtained
\end{abstract}

\section{W. Li $(\bowtie) \cdot$ C. Shi}

College of Civil Engineering, Hunan University,

Changsha 410082, Hunan, China

e-mail: wengui.li@hnu.edu.cn

W. Li · S. Kawashima - D. J. Corr · S. P. Shah

Center for Advanced Cement-Based Materials,

Northwestern University, Evanston, IL 60208, USA

e-mail: s-shah@northwestern.edu

W. Li · J. Xiao

Department of Structural Engineering, Tongji University, Shanghai 200092, China

\section{S. Kawashima}

Department of Civil Engineering and Engineering Mechanics, Columbia University, New York, NY 10027, USA using peak-force QNM were consistent with those obtained by modulus mapping, while the different in modulus probability distribution could be related to the different nanomechancial theories and contact forces. The probability distributions of elastic modulus measured using nanomechanical methods to provide a basis for the different types of phases existing in cement paste. Based on the observation with high spatial resolution, cement paste can be likely found as nanocalse granular material, in which different submicron scale or basic nanoscale grain units pack together. It indicates that the peak-force QNM can effectively provide an effective insight into the nanostructure characteristic and corresponding nanomechanical properties of cement paste.

Keywords Hardened cement paste .

Nanoindentation · Modulus mapping $\cdot$ Peak-force quantitative nanomechanical mapping (QNM) . Nanomechanical properties

\section{Introduction}

There is a fundamental need for the quantitative characterization of the nanomechanical properties for cement-based materials. This is essential not only for a better understanding of material behavior, but also for providing a basis for the design and preparation of novel cement composites with well-defined properties 
for specific structural applications [1, 2]. For instance, it suggests that a binder-mineral interface with high ductility and a significant stiffness contrast may improve significantly the fracture resistance of concrete. Various investigations such as scanning electron microscopy (SEM) and atomic force microscopy (AFM) are excellent methods to characterize the nano/microstructures of cement-based materials with the surface morphology [3, 4]. However, measuring local mechanical properties of cement-based materials at nanoscale requires nanomechanical probe-based methods. Investigations on the nanoscale properties have received much attention in recent years [5-7]. Recently, three advanced nanomechanical techniques have been developed including depth sensing nanoindentation [8-10], quantitative modulus mapping in the form of scanning probe microscopy (SPM) [11, 12] and AFM based peak-force quantitative nanomechanical mapping (QNM) [13, 14]. The capabilities of the three instruments cover a wide range of contact area and displacement resolution from sub-micron scale in the case of nanoindentation down to nanoscale in peak-force QNM.

The nanomechanical methods can be used to assess the nanomechanical properties and nanostructure of the individual constituents of heterogeneous materials and, in this way, to provide meaningful experimental data for the parameters of multi-scale numerical modeling. Nanoindentation is one of the most widely adopted techniques for this purpose of nanoscale characterization, and determines elastic modulus and hardness based on the measured indenting forcedisplacement curve. Among various methods to calculate the elastic modulus and hardness from the curve, Oliver and Pharr's method has been widely adopted because of its reliable/concise hypothesis [15, 16]. In contrast to nanoindentation tests, microindentation tests do not probe phase properties, but are found to reproduce composite properties, as shown and discussed for cement paste and for other composite materials in $[17,18]$. The modulus mapping technique employs aspects of nanoDMA and in situ SPM imaging to realize characterization capabilities to oscillate the indenter tip with small forces while monitoring the resultant displacement and phase lag due to material responses [11, 19]. In situ SPM imaging allows the indenter tip to be rastered across the material surface and is typically used to produce a topographic image of material surface. As for the modulus mapping technique, the advantage is that the contact stiffness can be obtained continuously during scanning using smaller contact force, which could be an ideal technique for multiphase and composite materials. The peak-force QNM is a new operating mode of AFM technique that can operate with a wide variety of standard AFM probes [13, 20]. In peak-force QNM, the probe is oscillated at a typical frequency of $2 \mathrm{kHz}$ (far below the resonance of the cantilever) with typical peak to peak amplitudes in air of $300 \mathrm{~nm}$. This brings the probe periodically into contact with the sample for a short period of time (less than $100 \mu \mathrm{s}$ ) and a periodically oscillating force is exerted onto the sample surface. Due to the high speed of modern AFM controllers, the individual force versus separation curves can be collected for each tap of the probe onto the sample surface. Peak-force QNM has been recognized as a useful method for the assessment of local mechanical properties of materials, which can also provide phase contrast maps for a qualitative characterization of materials with a very high spatial resolution [6].

The testing parameters of the three nanomechanical methods are different from each other. For these methods, it needs tip radius, probe stiffness, applied force, etc., which are set up according to the parameters in Refs. [5, 6, 12]. For both nanoindentation and modulus mapping, the applied force is needed as an input parameter before measurements. For modulus mapping, in addition to the applied force, the indent radius of curvature is also needed; specifically, its value can be determined during the calibration. As for peak-force QNM, the input parameters include tip radius, probe stiffness and deflection sensitivity. All the calibrations of the three nanomechanical methods are conducted with standard quartz [21].

The objective of this study is to comparatively investigate the nanomechanical properties of cement paste using three different methods (nanoindentation, modulus mapping and peak-force QNM). The relative merits of the application of these three methods are specifically discussed for the determination of elastic modulus of cement paste from $1-\mu \mathrm{m}$ length scale to tens of nanometers scale. It will provide an insight into the application of various nanomechanical methods as applied to cement-based materials. Due to the heterogeneity of cement paste, it is important to understand their basic mechanical behaviors at micro/nanoscale. 
Therefore, the nanomechanical properties of cement paste will be experimentally measured by the three nanomechanical methods, and these measurements will be used to help illuminate the discussion regarding the multiple modal distributions of nanomechanical properties in cement paste.

\section{Experimental program}

\subsection{Nanomechnical devices}

A static nanoindenation experiment was performed with a nanomechanical testing system (Hysitron) equipped with an in-place imaging mode $[5,8]$. The nanoindentation instrument consists of a force-displacement transducer with electrostatic force actuation and displacement sensing electronics. A twodimensional Berkovich indenter with a high elastic modulus is used. The system is calibrated with standard quartz crystal before performing the actual indentation on the sample to ensure that the probe is not contaminated or damaged.

Quantitative modulus mapping in the form of SPM images is acquired using the direct force modulation (nanoDMA) operating mode of the nanomechanical testing system $[11,12]$. A cubecorner diamond tip is attached to a force-displacement transducer. As with nanoindentation, the system was calibrated with a standard quartz crystal. A lockin amplifier is used to analyze the sample response, yielding displacement amplitude, and the phase shift between the contact force and the displacement. The contact stiffness and material damping are calculated from the amplitude and phase shift using a dynamic model.
Peak-force QNM is a mode with improved force resolution combined with real time calculation of elastic modulus at each surface contact, which can detect local elastic modulus $[6,13]$. This technique (Bruker AXS) can provide compositional mapping of a complex composite material while providing high resolution. In peak-force tapping, the probe and sample are intermittently brought together (similar to tapping mode) to contact the surface for a short period, which eliminates lateral forces. Unlike tapping mode, where the feedback loop keeps the cantilever vibration amplitude as a constant, peak-force tapping controls the maximum force on the tip. This protects the tip and sample from damage while allowing the tip sample contact area to be minimized. The testing parameters of the three techniques are listed in Table 1.

\subsection{Sample preparation}

There are three primary goals of the sample preparation procedure for the three nanomechanical methods, including achieving as flat a surface as possible, obtaining repeatable results, and minimizing the sample disturbance $[22,24]$. The procedure described here is optimized to satisfy these three goals. In the first step, a $5 \mathrm{~mm}$ thick $10 \mathrm{~mm} \times 10 \mathrm{~mm}$ block of hardened cement paste $(\mathrm{w} / \mathrm{c}=0.40)$ was produced from type I Portland cement (see Table 2) and water cured under $20{ }^{\circ} \mathrm{C}$ and $95 \%$ relative humidity condition. At an age of 90 days, the resulting cement paste cube with a size of $5 \mathrm{~mm}$ was impregnated with epoxy. After the epoxy impregnation, a small cylindrical sample $20 \mathrm{~mm}$ in diameter with the cement paste residing inside was prepared. Subsequently, the small cylindrical sample was then affixed with cyanoacrylate glue to a circular steel disc $15 \mathrm{~mm}$ in diameter.

Table 1 Testing parameters of the three techniques

\begin{tabular}{llll}
\hline & Nanoindentation & Modulus mapping & PeakForce QNM \\
\hline Probe type & Berkovich (2D 142.3 $)$ & Berkovich (1D cube-corner) & AFM cantilever \\
Tip radius & $600 \mathrm{~nm}$ & - & $5 \mathrm{~nm}$ \\
Tip material & Diamond & Diamond & Diamond \\
Indent depth $(\mathrm{nm})$ & $200-800$ & $7-12$ & $2-8$ \\
Radius of curvature & - & $557.14 \mathrm{~nm}$ & - \\
Applied force & $1200 \mu \mathrm{N}$ & $20 \mu \mathrm{N}$ & $50 \mathrm{nN}$ \\
Deflection sensitivity & - & - & $70 \mathrm{~nm} / \mathrm{V}$ \\
Maximum resolution $(\mathrm{nm})$ & 800 & 60 & 5 \\
\hline
\end{tabular}


Table 2 Physiochemical compositions of Portland cement

\begin{tabular}{ll}
\hline Materials & Type I Portland cement \\
\hline $\mathrm{SiO}_{2}$ & 20.2 \\
$\mathrm{Al}_{2} \mathrm{O}_{3}$ & 4.7 \\
$\mathrm{Fe}_{2} \mathrm{O}_{3}$ & 3.3 \\
$\mathrm{SO}_{3}$ & 3.3 \\
$\mathrm{CaO}$ & 62.9 \\
$\mathrm{MgO}$ & 2.7 \\
$\mathrm{Na}$ & $\mathrm{O}$ \\
$\mathrm{K}_{2} \mathrm{O}$ & - \\
LOI & - \\
Total & 1.1 \\
Density $\left(\mathrm{g} / \mathrm{cm}^{3}\right)$ & 98.2 \\
\hline
\end{tabular}

The exposed flat surface of the cement paste sample was ground and polished using the similar techniques presented in Refs. [5, 23, 24], which has been proven to be useful and efficient for sample preparation of cement-based materials.

The completed polishing surface is shown in Fig. 1a. It should be noted that the focus of the sample preparation was to achieve a flat surface that would, at the same time, be easily accessible to three kinds of techniques. Figure $1 \mathrm{~b}$ shows the surface topography of a porous square region of the polished sample acquired by high resolution tapping mode AFM. As expected, the root-mean-square surface roughness of sample based on the topography map is $93.9 \pm 20 \mathrm{~nm}$ for scanning size of $50 \mu \mathrm{m} \times 50 \mu \mathrm{m}$, which is sufficiently low enough for nanoindentation, modulus mapping and peak-force QNM testing [6, 20, 22]. The three nanomechanical methods are applied to measure the nanomechanical properties on the same location of the sample surface, respectively. As the sample surface could potentially be damaged by the larger force in static nanoindentation, thus peak-force QNM and modulus mapping were performed always first on the sample compared with nanoindentation testing.

As mentioned earlier, the modulus mapping and peak-force QNM were performed on two devices (Hysitron and Bruker AXS, respectively). For this reason, when switching peak-force QNM testing to modulus mapping from one method to the other, it is necessary to fully confirm that the measurements are each performed at the same position on the same sample. To achieve this, extra high resolution images were captured, and image analysis was applied to measure the distance and adjust the test position and location distance to make sure all measurements are conducted at the same region of the tested sample. As for the tested area, the deviation should be acceptable less than $0.5 \mu \mathrm{m}$.

\section{Measurements and results}

\subsection{Nanoindentation}

The nanoindentation was performed on cement paste sample by single loading with a maximum load of $1200 \mu \mathrm{N}$, which is similar to those presented in $[5,23$, 24]. The completed polished surface is shown in Fig. 2, which indicates surface topography of a square region of the tested sample. The distance between indents was set at $3 \mu \mathrm{m}$, in order to avoid possible overlapping of plastic deformation zone onto neighboring indents. In order to achieve a more precise profile, two different regions were indented to cover more phases for statistical analysis.
Fig. 1 Cement paste sample after polishing. a AFM image, b surface topography $(50 \mu \mathrm{m} \times 50$ $\mu \mathrm{m}$ )

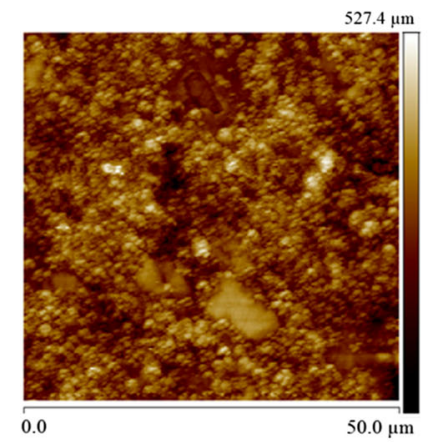

(a)

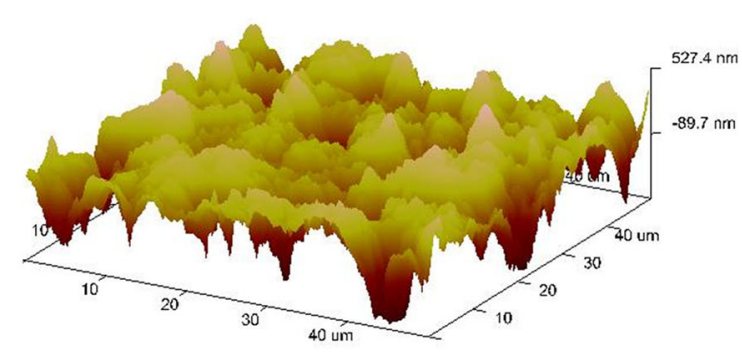

(b) 
Fig. 2 Cement paste sample after polishing. a Optical image, b indent location

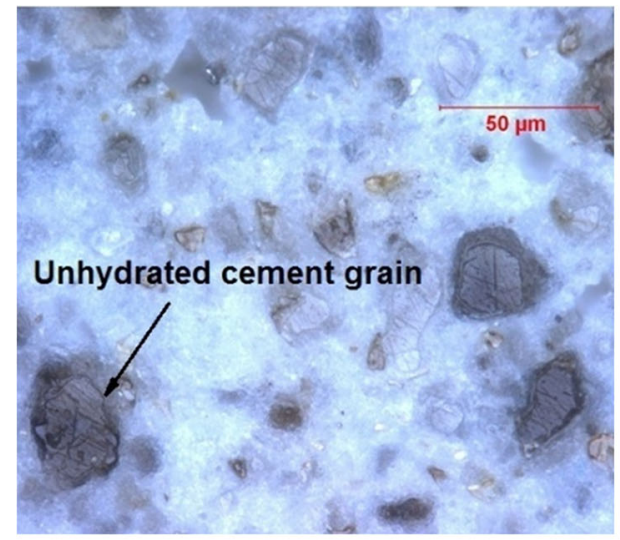

(a)

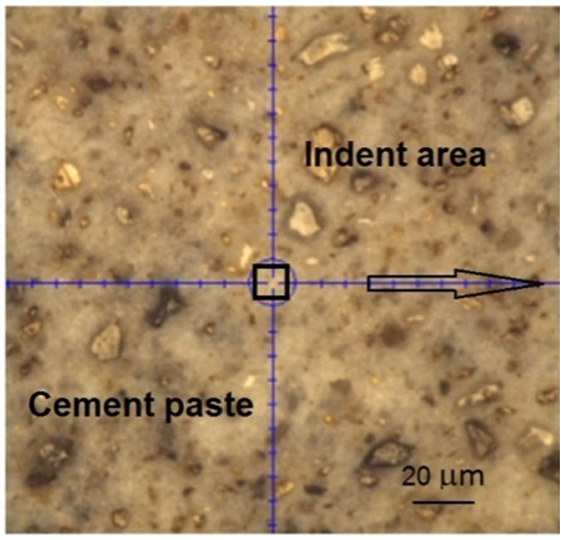

(b)
As identified by the past works [25-29], the areas with relatively high indentation modulus value (higher than $50.0 \mathrm{GPa}$ ) are ascribed to the existence of unhydrated, or partially hydrated cement particles, and the regions that have indentation modulus values ranging from 10.0 to $50.0 \mathrm{GPa}$ are hydration products. Moreover, the lower values of indentation modulus (less than $10 \mathrm{GPa}$ ) are predominantly due to the porous regions in cement paste. Based on the nanoindentation measurements results, different representative indent load-depth cures which located in different modulus distribution ranges were shown correspondingly. Figure 3 shows typical loading and unloading curves during indentations on different phases of cement paste, including porosity, hydrates (predominantly calcium silicate hydrate and others such as slight

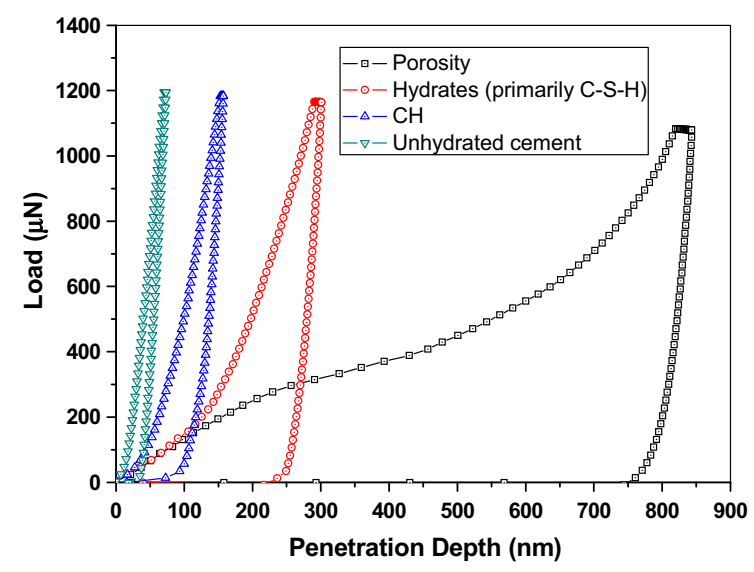

Fig. 3 Representative load-depth curves of different phases in cement paste ettringite), calcium hydroxide ( $\mathrm{CH})$, and unhydrated cement grains. Each load/displacement diagram was plotted and inspected for signs of problems due to surface roughness, such as abnormal or discontinuous shapes. The grid indentation method was performed large number of indents on the sample, and statistical analysis of these data obtained from all the indentations provides information about the overall nanomechanical properties of the sample. Figures 4 and 5 are contour maps and statistical analysis of indentation modulus for the areas of $100 \mu \mathrm{m} \times 100 \mu \mathrm{m}$ and 15 $\mu \mathrm{m} \times 15 \mu \mathrm{m}$, respectively. Results indicate that the general quantitative correlation between the mechanical properties and phase features can be obverted clearly. It shows that the peak value of the modulus probability plot for the area of $100 \mu \mathrm{m} \times 100 \mu \mathrm{m}$ falls between 10.0 and $15.0 \mathrm{GPa}$, while the peak value of modulus probability plot for the area of $15 \mu \mathrm{m} \times 15$ $\mu \mathrm{m}$ is between 15 and $20 \mathrm{GPa}$. From the probability distribution, it is found that the peak value of the modulus probability plot in the area of $15 \mu \mathrm{m} \times 15$ $\mu \mathrm{m}$ is visually higher than that in the area of 100 $\mu \mathrm{m} \times 100 \mu \mathrm{m}$. This indicates that more volume fraction of hydration products such $\mathrm{C}-\mathrm{S}-\mathrm{H}$ phase exist in the smaller indent area. Moreover, the modulus distributions that correspond to the porosity low than $10.0 \mathrm{GPa}$ and cement clinker above $50.0 \mathrm{GPa}$ are significantly increased for the larger indent area, resulting in a greater indentation modulus distribution range and variation correspondingly. The illustration of cumulative probability of cement paste for the areas of $100 \mu \mathrm{m} \times 100 \mu \mathrm{m}$ and $15 \mu \mathrm{m} \times 15 \mu \mathrm{m}$ are shown in Fig. 6. It indicates that modulus results ranging 


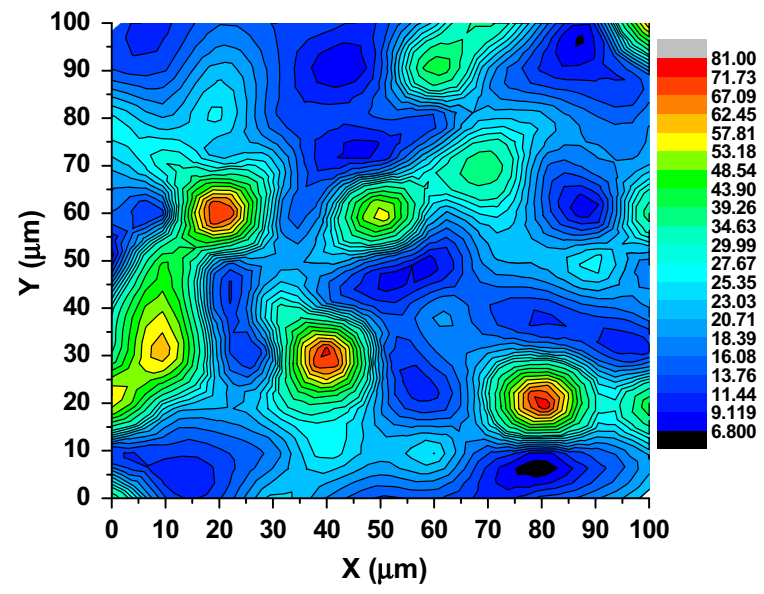

(a)

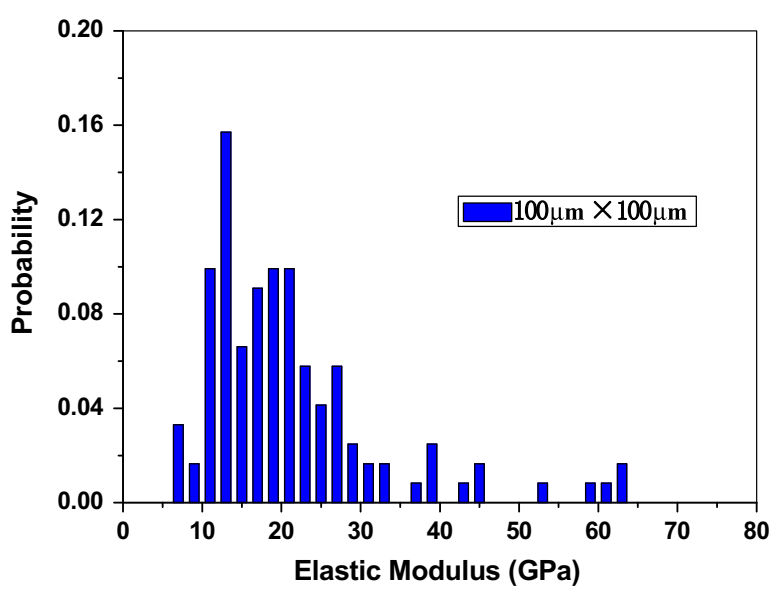

(b)

Fig. 4 Elastic modulus distribution obtained by nanoindentation (area of $100 \mu \mathrm{m} \times 100 \mu \mathrm{m}$ ). a Indentation modulus contour map, b probability distribution (bin size $=2.0 \mathrm{GPa}$ )

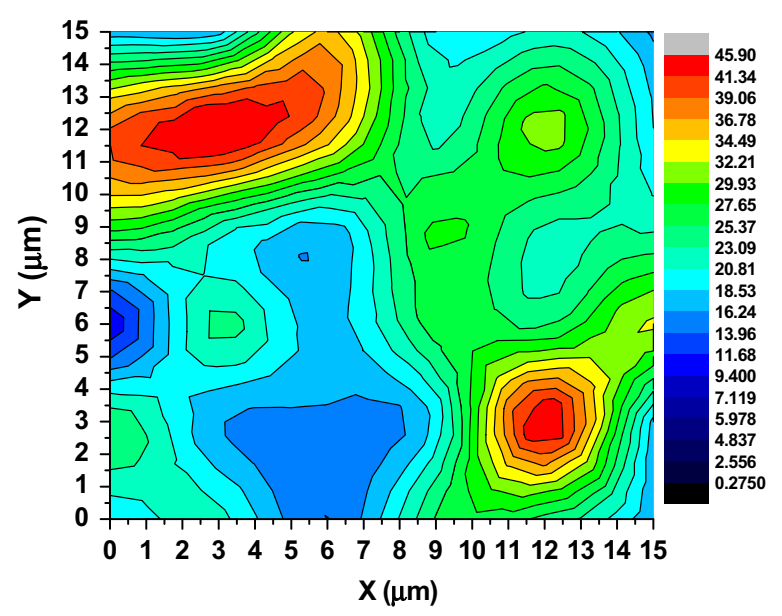

(a)

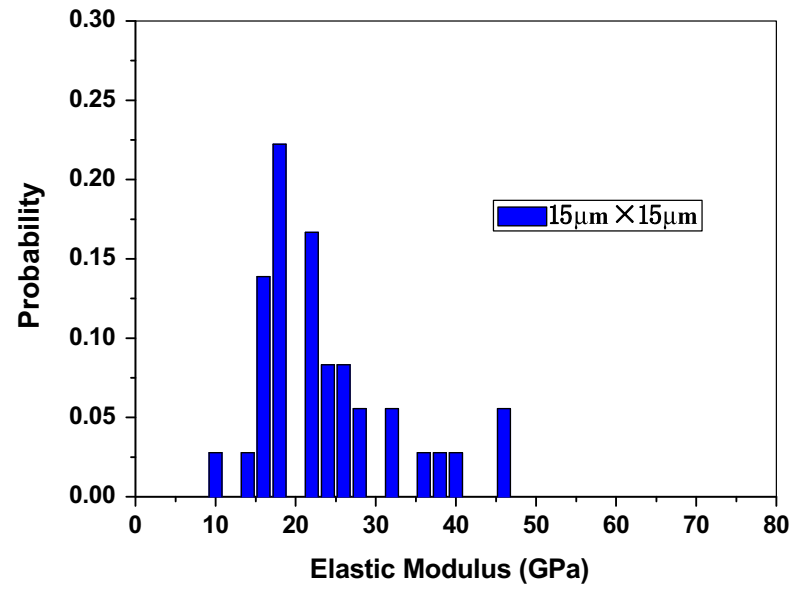

(b)

Fig. 5 Elastic modulus distribution obtained by nanoindentation (area of $15 \mu \mathrm{m} \times 15 \mu \mathrm{m}$ ). a Indentation modulus contour map, b probability distribution (bin size $=2.0 \mathrm{GPa}$ )

from 10 to $50 \mathrm{GPa}$ are representative of hydration phases, and the main effect on the volume fractions of the different phases presented by nanoindentation in the hardened cement paste in the measurement area [5, 26]. Although many studies have been conducted on the nanomechanical properties of cement paste, little has been reported to characterize and provide quantitative measurements considering different sizes of investigated area. From the measurements, the basic distribution range and volume fraction of the phases in cement paste are obviously influenced by the tested areas, and the nanoindentation measurements should further consider the different sizes of investigated areas.

\subsection{Modulus mapping}

The modulus mapping was further performed to study the nanomechanical properties with higher resolution compared to static nanoindentation [12, 19]. When switching the modulus mapping testing mode to nanoindentation mode to study the nanomechanical 


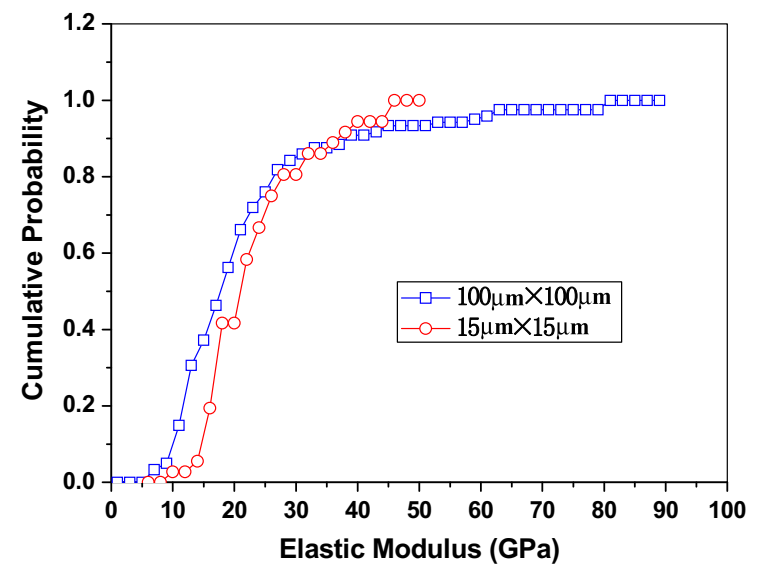

Fig. 6 Cumulative probability distribution of elastic modulus obtained by nanoindentation

properties of cement paste, the position of the tested sample remains unchanged. Eventually, it is efficient to conduct reliable comparison between the three different method measurements on the same region of the same sample.

Figure 7 presents the elastic modulus contour maps and statistical analysis of the area of $15 \mu \mathrm{m} \times 15 \mu \mathrm{m}$. The range of measured elastic modulus is found to be significantly greater than that obtained by nanoindentation. It can be attributed to the higher resolution and smaller contact area of modulus mapping compared with nanoindentation. The results of modulus mapping for the scanned area $5 \mu \mathrm{m} \times 5 \mu \mathrm{m}$ are shown in Fig. 8 . It is found that the quantitative modulus variations in modulus mapping are larger than those in nanoindentation. It reveals that the modulus mapping technique is more sensitive than nanoindentation with a lower contact force. In general, obvious local elastic modulus variations were observed at the sample surface which comprised different phases and interfaces between hydration products such as inner and outer hydration products. Previous workers also have identified variations in elastic modulus as being due to different phases in the cement $[8,26]$. On the other hand, clear abrupt changes in the elastic modulus could be due to the interfaces between the porous areas and different hydration products. The peak of the modulus probability plot for the area of $15 \mu \mathrm{m} \times 15$ $\mu \mathrm{m}$ falls between 15 and $20 \mathrm{GPa}$, while the peak of modulus probability plot for the area of $5 \mu \mathrm{m} \times 5 \mu \mathrm{m}$ is between 20 and $25 \mathrm{GPa}$. More importantly, it seems that the peak value of elastic modulus in the probability plot in the area of $5 \mu \mathrm{m} \times 5 \mu \mathrm{m}$ is a somewhat higher than that in area of $15 \mu \mathrm{m} \times 15 \mu \mathrm{m}$. Moreover, it is needed to note that it is likely for the probability distributions of elastic modulus measured using modulus mapping to provide an obvious indication for the different types of hydration products. However, probability distribution of elastic modulus can't exactly indicate how many different types of C$\mathrm{S}-\mathrm{H}$ exist in cement paste such as high density and low density $\mathrm{C}-\mathrm{S}-\mathrm{H}[6,7]$. The difference in the cumulative probability distribution of phases between the different measured area of $15 \mu \mathrm{m} \times 15 \mu \mathrm{m}$ and the area of 5 $\mu \mathrm{m} \times 5 \mu \mathrm{m}$ is typically consistent, as shown in Fig. 9 .

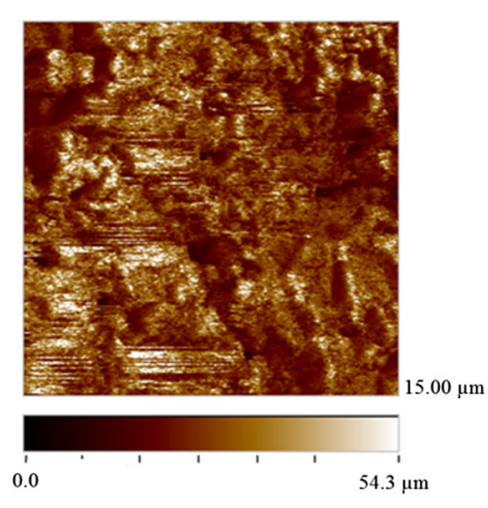

(a)

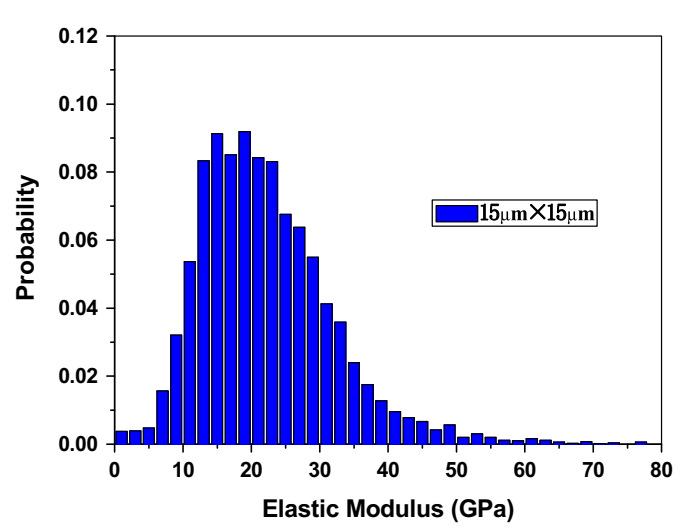

(b)

Fig. 7 Elastic modulus obtained by modulus mapping (area of $15 \mu \mathrm{m} \times 15 \mu \mathrm{m}$ ). a Elastic modulus contour map, b probability distribution (bin size $=2.0 \mathrm{GPa})$ 


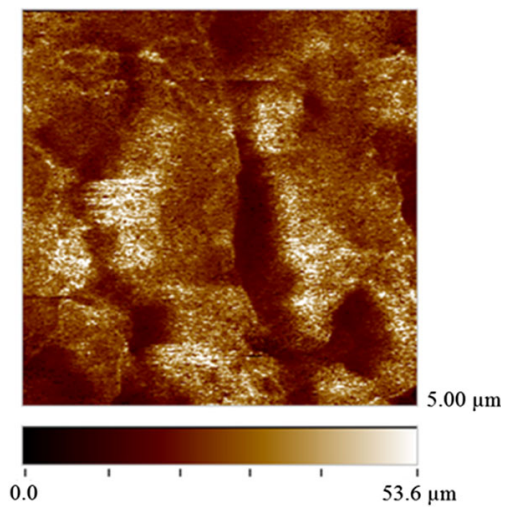

(a)

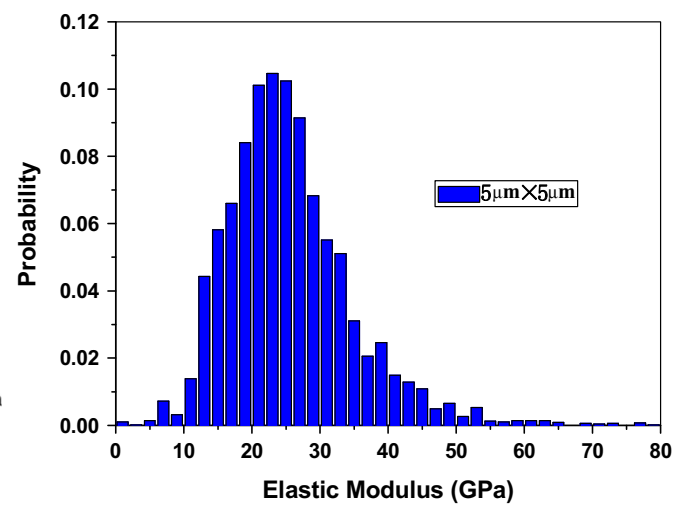

(b)

Fig. 8 Elastic modulus distribution obtained by modulus mapping (area of $5 \mu \mathrm{m} \times 5 \mu \mathrm{m}$ ). a Elastic modulus contour map, b probability distribution (bin size $=2.0 \mathrm{GPa}$ )

Assuming that the $\mathrm{C}-\mathrm{S}-\mathrm{H}$ phase can be identified with two characteristic forms, low density $\mathrm{C}-\mathrm{S}-\mathrm{H}$ and high density $\mathrm{C}-\mathrm{S}-\mathrm{H}[26,30]$, the area of $5 \mu \mathrm{m} \times 5 \mu \mathrm{m}$ is found to have a smaller volume fraction of low density $\mathrm{C}-\mathrm{S}-\mathrm{H}$, compared to the area of $15 \mu \mathrm{m} \times 15 \mu \mathrm{m}$.

\subsection{Peak-force QNM}

In addition to nanoindentation and modulus mapping, peak-force QNM technique was used to evaluate nanomechanical properties at a very high resolution (5-10 nm). When switching peak-force QNM testing to modulus mapping from one method to the other, it is necessary to fully confirm that the measurements are each performed at the same position on the same

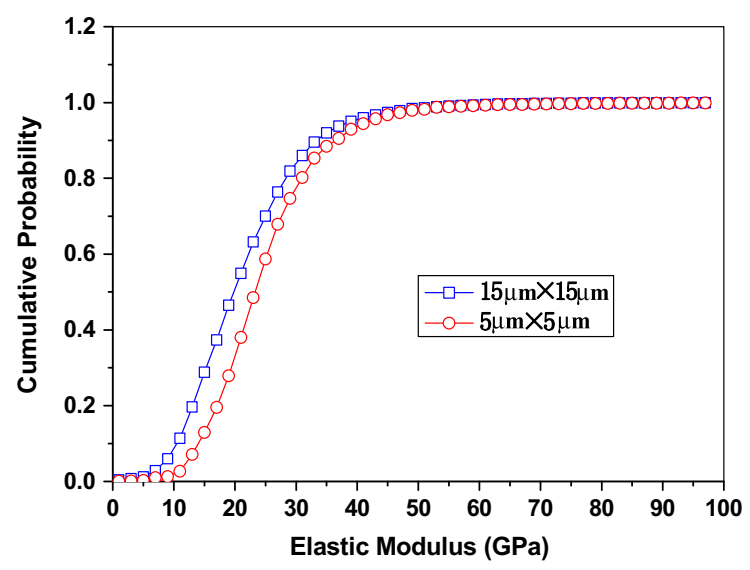

Fig. 9 Cumulative probability distribution of elastic modulus obtained by modulus mapping sample. To achieve this, extra high resolution images with distinguished features were captured, and image analysis was applied to measure the distance and adjust the test position based on a precise distance and direction from the features to make sure all measurements are conducted at the same region of the tested sample.

Based on the elastic modulus contour image of cement paste offered in Fig. 10, visual nanostructural characteristics of the area of $5 \mu \mathrm{m} \times 5 \mu \mathrm{m}$ can be obtained. There is a significant difference observed in modulus distributions between the multi-phases in cement paste. The porosity and the interfaces between nanoscale grains with a lower elastic modulus appear darker, while the calcium hydroxides, unhydrated cement particles and other phases with higher elastic modulus exhibit brighter. In order to analyze the nanostructure and nanomechanical properties in more details, the results of smaller area of $1 \mu \mathrm{m} \times 1 \mu \mathrm{m}$ were presented in Fig. 11. The elastic modulus contour maps also demonstrate that the cement paste is a granular material, where the sub-micron scale grains or basic nanoscale units pack together densely, which has been also been reported in other researches $[31,32]$. Based on the basic composition of the selected cement, the hydration products should be predominantly composed of $\mathrm{C}-\mathrm{S}-\mathrm{H}$ grains. Hence, the discussion was focused on the aforementioned $\mathrm{C}-\mathrm{S}-\mathrm{H}$ grains and the shape characteristics of them. Compared to the different scanned areas with peak-force QNM, the elastic modulus distribution range of the area of $5 \mu \mathrm{m} \times 5 \mu \mathrm{m}$ is slightly larger than that of the 


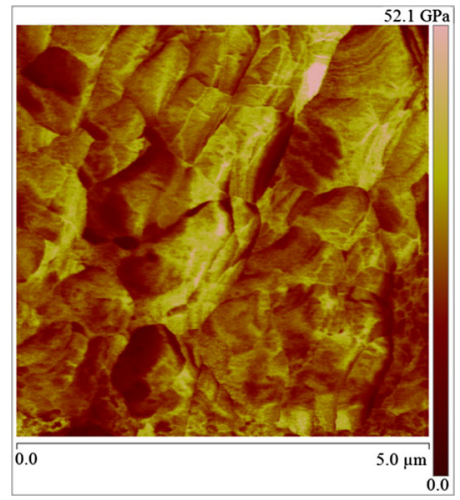

(a)

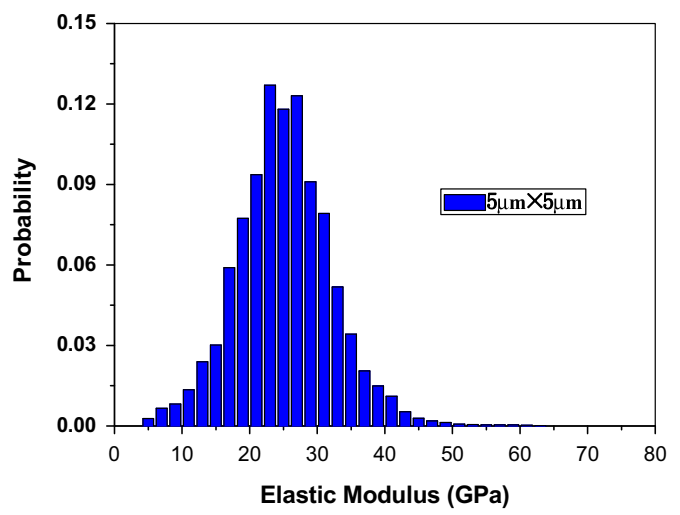

(b)

Fig. 10 Elastic modulus distribution obtained by peak-force QNM (area of $5 \mu \mathrm{m} \times 5 \mu \mathrm{m}$ ). a Elastic modulus contour map, b probability distribution (bin size $=2.0 \mathrm{GPa}$ )

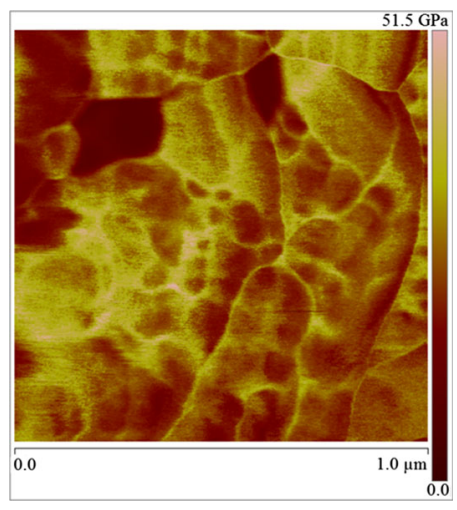

(a)

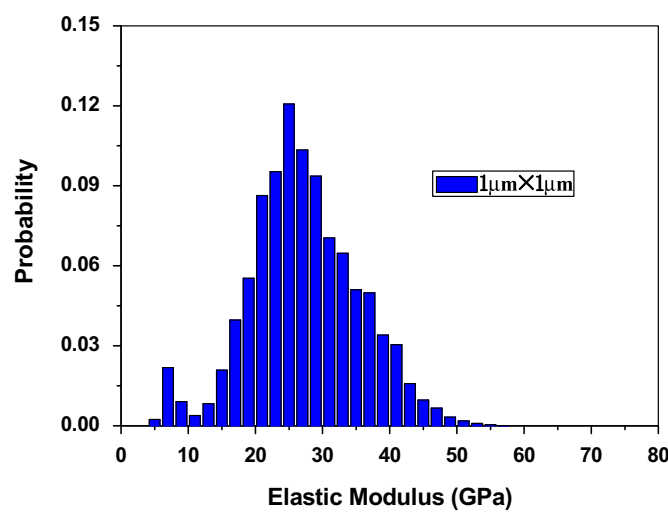

(b)

Fig. 11 Elastic modulus distribution obtained by peak-force QNM (area of $1 \mu \mathrm{m} \times 1 \mu \mathrm{m}$ ). a Elastic modulus contour map, b probability distribution (bin size $=2.0 \mathrm{GPa}$ )

area of $1 \mu \mathrm{m} \times 1 \mu \mathrm{m}$ because of the different nanoscale grains experiencing different intrinsic elastic modulus, respectively. The probability distributions of elastic modulus for cement paste seems to provide a indication for the occurrence of different types of C-S-H, but can't exactly distinguish present there are how many types of $\mathrm{C}-\mathrm{S}-\mathrm{H}$ which are representative of low density and high density $\mathrm{C}-\mathrm{S}-\mathrm{H}$, as reported in previous researches [9, 17]. When comparing the analogous probability distribution results of the two different scanning areas, the peak value of the modulus probability plot for area of 5 $\mu \mathrm{m} \times 5 \mu \mathrm{m}$ appears to be consistent with that of 1 $\mu \mathrm{m} \times 1 \mu \mathrm{m}$, which is found to around 20-25 GPa. It indicates that the measurement result of area of 5 $\mu \mathrm{m} \times 5 \mu \mathrm{m}$ has a similar cumulative probability distribution with the area of $1 \mu \mathrm{m} \times 1 \mu \mathrm{m}$, as shown in Fig. 12. Furthermore, it can be found that the phase volumes fractions in cement paste typically keep constant with each other for different scanning areas using peak-force QNM testing.

\section{Comparison analysis}

\subsection{Nanoindentation and modulus mapping}

Compared to nanoindentation, the modulus mapping allows for evaluation of local mechanical properties over a smaller area with higher spatial resolution at 


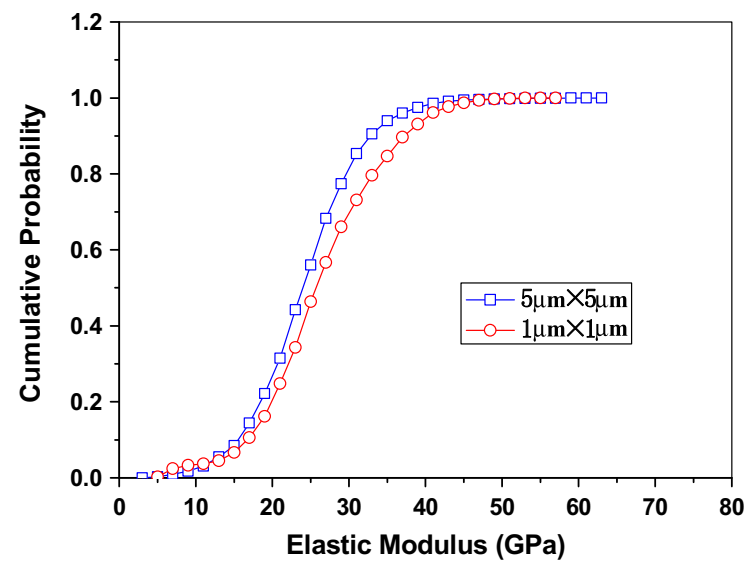

Fig. 12 Cumulative probability distribution of elastic modulus obtained by peak-force QNM

nanoscale $[16,33]$. In nanoindentation, although the nanomechanical properties of different phases in cement paste can be estimated by statistical analysis, the separation between indentations is required to be large enough to avoid the effects of residual stress and interaction between the neighboring indents on the results. For Berkovich diamond tips, the lateral dimension of the indentation is greater than the depth of indentation. Therefore, to avoid error in elastic modulus due to possible interaction between adjacent indentions, a separation of 5-10 times the lateral indentation dimensions is employed as a general guide, which means a minimum separation of $3 \mu \mathrm{m}$ for nanoindentation testing. As a result, determination of the local quantitative variation at the nanometer level is practically impossible for nanoindentation, however, the modulus mapping technique in the form of SPM images can overcome these shortcomings [8]. This is because the sample under the tip are probed at a shallow indent depth (elastic deformation), and once the tip force is removed, the probed materials can fully recover without local residual stress or permanent deformation. Moreover, the modulus mapping can provide measurements on independent phases with greater precision.

From Fig. 13a, the elastic modulus distribution obtained by modulus mapping is generally consistent with that by nanoindentation at the same area of 15 $\mu \mathrm{m} \times 15 \mu \mathrm{m}$. However, the average nanoindentation modulus was around $23.8 \pm 8.7 \mathrm{GPa}$, while the average modulus by modulus mapping was around $22.2 \pm 9.8$ GPa. Generally, the average elastic modulus measured by nanoindentation is consistent with those measured by modulus mapping. Nonetheless visually, the a little bit higher average modulus value in nanoindentation could be a result of the local confinement effect and interaction between multiple phases in the indent area, because the indent depth of nanoindentation is much higher than the probed depth in modulus mapping. Furthermore, Fig. 13b shows that the peak value of the modulus probability plot extracted from nanoindentation is a somewhat higher than the corresponding peak value measured with modulus mapping. More importantly, the large indent area can reduce the influence of porosity, and unhydrated cement residuals in the nanoindentation measurement compared with modulus mapping measurement could explain the generally concentrated contribution in the measured elastic modulus by modulus mapping.

\subsection{Modulus mapping and peak-force QNM}

Compared to the contact radius $(60 \mathrm{~nm})$ of modulus mapping testing, the probe radius of peak-force QNM mode is significantly sharper ( $5 \mathrm{~nm}$ ), which makes the spatial resolution significantly higher $[6,7]$. For this reason, the peak-force QNM appears more useful when investigating local nanomechanical properties of a small area in more details. For peak-force QNM, a typical scan takes $10 \mathrm{~min}$, which is significantly faster than the modulus mapping. After a scan is completed, 512 lines of data are available with 512 data points in each line. On the other hand, compared to peak-force QNM mode, the modulus mapping will be more convenient for a larger measured area.

As indicated in Fig. 14a, except for the variation characterization, the modulus distribution of the same measuring area of $5 \mu \mathrm{m} \times 5 \mu \mathrm{m}$ obtained by peakforce QNM is typically consistent with those by modulus mapping. The average elastic modulus $(25.6 \pm 9.2 \mathrm{GPa})$ measured by modulus mapping is basically consistent with the average elastic modulus $(25.2 \pm 6.6 \mathrm{GPa})$ obtained by peak-force QNM. At the same time, the modulus probability characterization measured from peak-force QNM is similar to that measured with modulus mapping, which can be clearly seen in Fig. 14b. Furthermore, the peak value of the modulus probability plot measured by peakforce QNM is typically higher than the value obtained by modulus mapping. Consequently, the plausible 


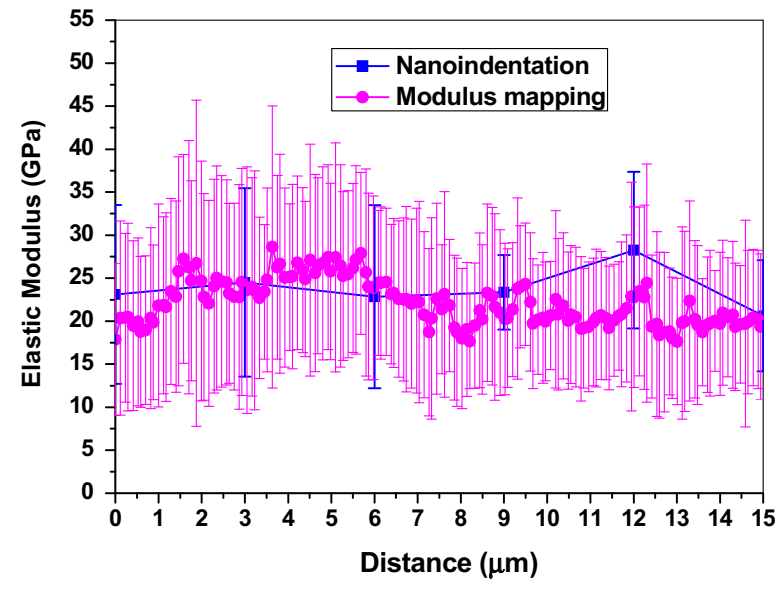

(a)

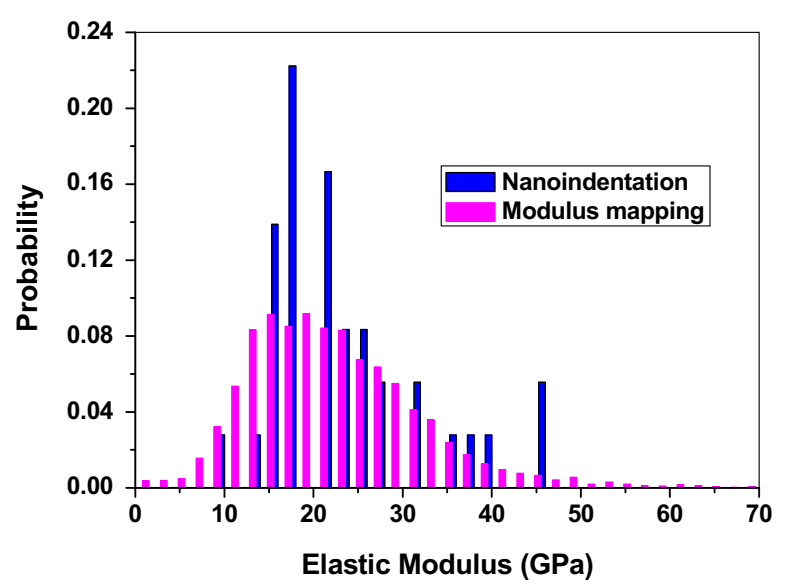

(b)

Fig. 13 Elastic modulus distribution of nanoindentation and modulus mapping (area of $15 \mu \mathrm{m} \times 15 \mu \mathrm{m}$ ). a Elastic modulus distribution, b probability distribution (bin size $=2.0 \mathrm{GPa}$ )

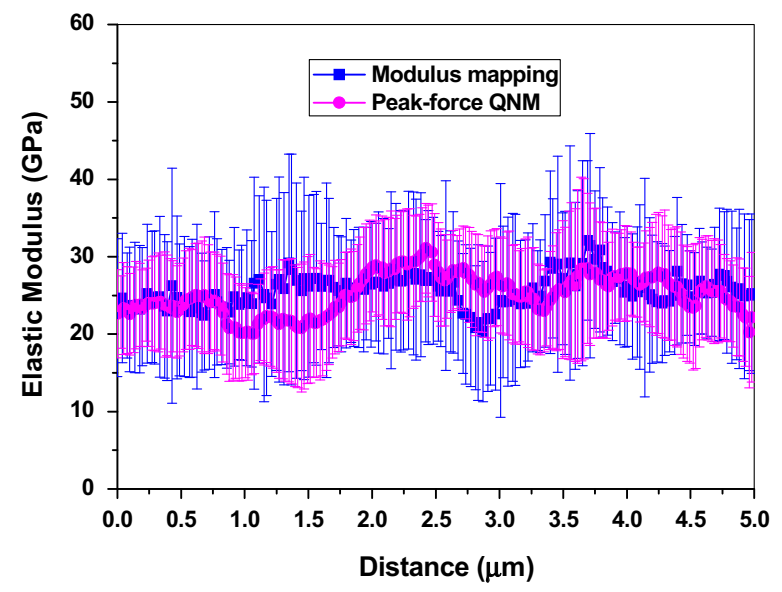

(a)

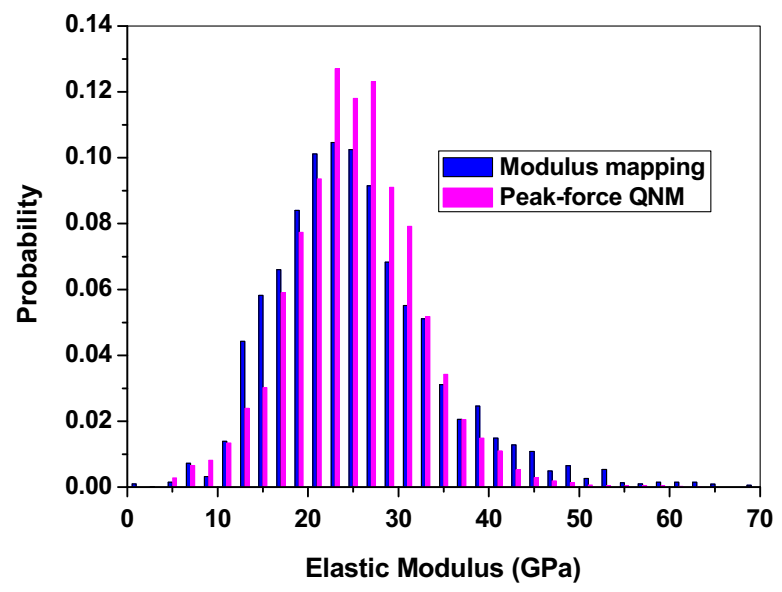

(b)

Fig. 14 Elastic modulus distributions of modulus mapping and peak-force QNM (area of $5 \mu \mathrm{m} \times 5 \mu \mathrm{m}$ ). a Elastic modulus distribution, b probability distribution (bin size $=2.0 \mathrm{GPa}$ )

explanation for this slight difference could be related to the different related nanomechancial theories and contact forces during testing for these two nanomechanical methods. Overall, it seems that the mean value and distribution characteristics of the measured values obtained by peak-force QNM are in good agreement with the measured results by the modulus mapping. Based on the probe radius of the peak-force QNM is quite sharper than that of modulus mapping (as shown in Table 1), the measured values are less likely to involve influence from the interaction between multiple phases and are thus likely more representative of the intrinsic properties of the $\mathrm{C}-\mathrm{S}-\mathrm{H}$ particle. In particular, the homogenized elastic modulus of the composite obtained from peak-force QNM measurements should present a relative smaller error when compared to the modulus mapping.

\subsection{Peak-force QNM and nanoindentation}

Based on the existing literature $[7,21,30]$, the $\mathrm{C}-\mathrm{S}-\mathrm{H}$ phase is the primary phase of the hydration products in 
cement paste, and the properties of cement paste are governed through the nucleation, packing, and stiffness of the nanoscale $\mathrm{C}-\mathrm{S}-\mathrm{H}$ particles. As the grains form at random sizes, improving the diversity in the size of nanoscale units can eventually lead to a denser and disorderly packing of the particles, which corresponds to a stronger and higher performance cement paste. The topography features of cement paste are of different sizes to the nanoscale grains hypothesized in the colloidal spheres model of granular particles, as shown in Fig. 15. It indicates that cement paste is a granular material, where the particles or basic nanoscale units pack together most orderly. When comparing the nanostructure characterization of cement paste from peak-force QNM measurement to the analogous results Refs. [34, 35], it demonstrates that the observed spatial variety of the grains packing fraction and grains shape can be associated with the diversity model of granular particles (predominately $\mathrm{C}-\mathrm{S}-\mathrm{H})$. On the other hand, the topographical image obtained from peak-force QNM has not only great intrinsic quantitative information on the gains size and shape but also insight into nanostructural morphology for cement paste.

The grain packing density considering a colloidal spheres structure in cement paste at nanoscale seems to provide a significant framework for reconciling the differences between the peak-force QNM measurements and those obtained by nanoindentation.
Therefore, it is hypothesized that the significant difference between the elastic modulus measured from peak-force QNM and those from nanoindentation is primarily due to the differences in grains packing at two different length scales (from one micrometer to tens of nanometers scale). With regard to the peak-force QNM testing which probes a much shallower depth than nanoindentation one, the peakforce QNM is likely more sensitive to surface grinding and polishing artifacts which seem to influence the packing density of the sample surface. During the sample preparation, the grinding and polishing may cause the nanoscale grains compaction of the sample surface. Such influences explain why the distribution range of elastic modulus measured by peak-force QNM in this study is found to be greater than those obtained from nanoindentation testing. It is hypothesized that the difference between the measured modulus obtained from nanoindentation and from peak-force QNM is also due primarily to differences in particle packing between the two length scales. One possibility should be noted that the increased grains packing is confined to the surface layer of sample due to the artifact of polishing process. As a results, whether the granular particles in cement paste are of colloidal sphere structure will still be a debate, as the granular particles may be formed from the layered model $\mathrm{C}-\mathrm{S}-\mathrm{H}$, which can be stacked together during the sample grinding and polishing [8, 34].

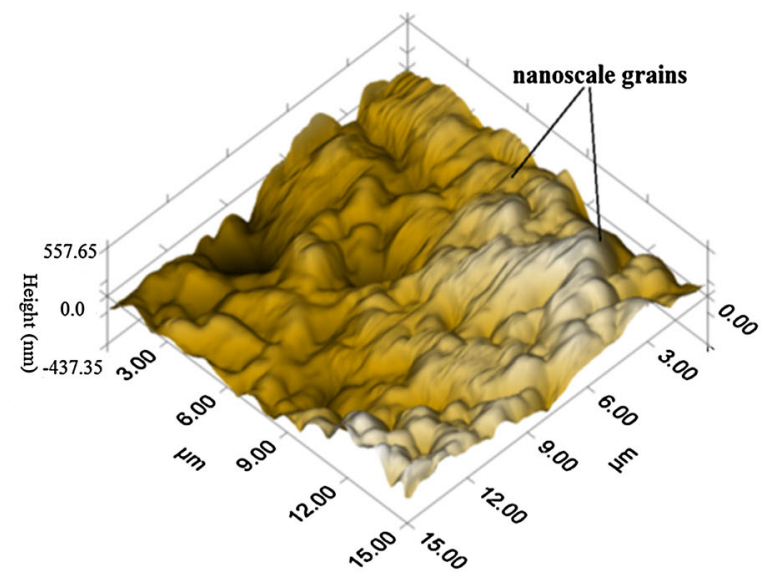

(a)

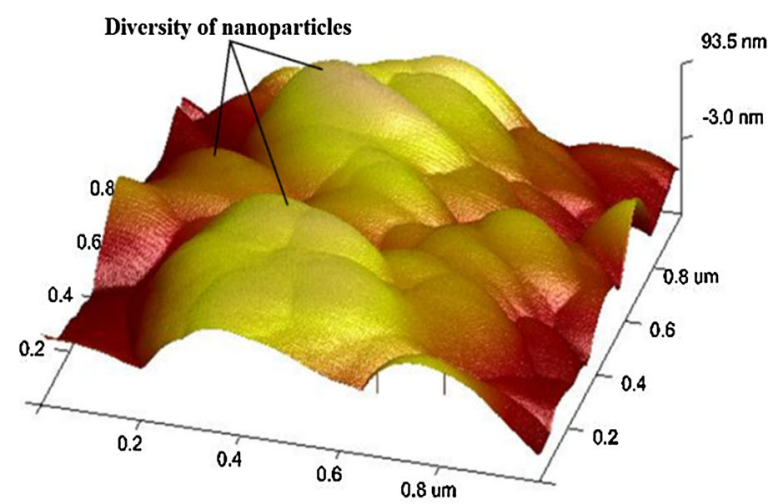

(b)

Fig. 15 Nanoscale grains characteristic of hardened cement paste (area of $15 \mu \mathrm{m} \times 15 \mu \mathrm{m}$ and $1 \mu \mathrm{m} \times 1 \mu \mathrm{m}$ ). a Topography by nanoindentation mapping, b topograghy by peak-force QNM 


\section{Conclusions}

The investigations on nanostructure and nanomechanical properties of cement paste were investigated using three correlative nanomechanical methods. Based on the analysis and comparisons on these different measurements, the following conclusions can be drawn:

(1) Compared with nanoindentation, modulus mapping and peak-force QNM allow for evaluating local mechanical properties at smaller measuring areas. The range of elastic modulus distribution measured by modulus mapping is relatively greater than that obtained from nanoindentation.

(2) The average value of elastic modulus measured by nanoindentation is generally consistent with those obtained using modulus mapping and peak-force QNM. The different modulus probability between them may be due to a result of the local confinement effect and interaction between multiple phases.

(3) The peak-force QNM can provide local mechanical properties in more details with a higher spatial resolution than modulus mapping. The peak value of modulus probability distribution plot measured by peak-force QNM is typically higher than the value obtained by modulus mapping, which could be related to the different related nanomechancial theories and contact forces during testing.

(4) Cement paste is likely found to be a granular material at nanocalse, in which the sub-micron scale or nanoscale grain units pack together. Moreover, compared with the nanoindentation, the peak-force QNM measurements can provide more insight into the characteristics of nanostructures and nanoscale granular particles for cement paste.

Acknowledgments The authors would like to gratefully acknowledge the National Natural Science Foundation of China (51408210), the Fundamental Research Funds for the Central Universities in Hunan University, China (531107040800) and the financial support from Infrastructure Technology Institute (ITI) at Northwestern University, USA under Grant DTRT06-G-0015. The first author is also grateful for the financial support of the Australian Research Council (DE150101751).

\section{References}

1. Sobolev K, Shah SP. Nanotechnology of concrete: recent developments and future perspectives, American Concrete Institute, Detroit, SP-254, 2008

2. Scrivener KL, Kirkpatrick RJ (2008) Innovation in use and research on cementitious material. Cem Concr Res 38(2):128-136

3. Sanchez F, Sobolev K (2010) Nanotechnology in concrete-a review. Constr Build Mater 24:2060-2071

4. Šavija B, Luković M, Hosseini SAS, Pacheco J, Schlangen E (2014) Corrosion induced cover cracking studied by $\mathrm{X}$-ray computed tomography, nanoindentation, and energy dispersive X-ray spectrometry (EDS). Mater Struct. doi:10. 1617/s11527-014-0292-9

5. Mondal P, Shah SP, Marks LD (2008) Nanoscale characterization of cementitious materials. ACI Mater J 105(2):174-179

6. Trtik P, Kaufmann J, Volz U (2012) On the use of peakforce tapping atomic force microscopy for quantification of the local elastic modulus in hardened cement paste. Cem Concr Res 42(1):215-221

7. Jones CA, Grasley ZC, Ohlhausen JA (2012) Measurement of elastic properties of calcium silicate hydrate with atomic force microscopy. Cem Concr Compos 34(4):468-477

8. Davydov D, Jirásek M, Kopecký L (2011) Critical aspects of nano-indentation technique in application to hardened cement paste. Cem Concr Res 41(1):20-29

9. Sorelli L, Constantinides G, Ulm F-J, Toutlemonde F (2008) The nano-mechanical signature of ultra high performance concrete by statistical nanoindentation techniques. Cem Concr Res 38(12):1447-1456

10. Wang XH, Jacobsen S, Lee SF, He JY, Zhang ZL (2010) Effect of silica fume, steel fiber and ITZ on the strength and fracture behavior of mortar. Mater Struct 43(1-2):125-139

11. Syed Asif SA, Wahl KJ, Colton RJ, Warren OL (2001) Quantitative imaging of nanoscale mechanical properties using hybrid nanoindentation and force modulation. J Appl Phys 90(3): 1192-1200

12. Balooch G, Marshall GW, Marshall SH, Warren OL, Asif SAS, Balooch M (2004) Evaluation of a new modulus mapping technique to investigate microstructural features of human teeth. J Biomech 37(8):1223-1232

13. Young TJ, Monclus MA, Burnett TL, Broughton WR, Ogin SL, Smith PA (2011) The use of the PeakForce ${ }^{\mathrm{TM}}$ quantitative nanomechanical mapping AFM-based method for high-resolution Young's modulus measurement of polymers. Meas Sci Technol 22(12):125703

14. Sahin O, Erina N (2008) High-resolution and large dynamic range nanomechanical mapping in tapping-mode atomic force microscopy. Nanotechnology 19(44):445717

15. Oliver WC, Pharr GM (1992) Improved technique for determining hardness and elastic modulus using load and displacement sensing indentation experiments. J Mater Res 7(6):1564-1583

16. Constantinides G, Ulm F, Van Vliet K (2003) On the use of nanoindentation for cementitious materials. Mater Struct 36(3):191-196

17. Constantinides G, Ulm F-J (2007) The nanogranular nature of C-S-H. J Mech Phys Solids 55(1):64-90 
18. Randall NX, Vandamme M, Ulm F-J (2009) Nanoindentation analysis as a two dimensional tool for mapping the mechanical properties of complex surfaces. J Mater Res 24(3):679-690

19. Uskokovic PS, Tang CY, Tsui CP, Ignjatovic N, Uskokovic DP (2007) Micromechanical properties of a hydroxyapatite/ poly-L-lactide biocomposite using nanoindentation and modulus mapping. J Eur Ceram Soc 27(2-3):1559-1564

20. Vanlandingham MR, McKnight SH, Palmese GR, Elings JR, Huang X, Bogetti TA, Eduljee RF, Gillespie J (1997) Nanoscale indentation of polymer systems using the atomic force microscope. J Adhes 64(1-4):31-59

21. Li WG, Xiao JZ, Kawashma S, Shekhawat GS, Shah SP (2014) Experimental investigation on quantitative nanomechanical properties of cement paste. ACI Mater J 111(1-6):603-612

22. Miller M, Bobko C, Vandamme M, Ulm F-J (2008) Surface roughness criteria for cement paste nanoindentation. Cem Concr Res 38(4):467-476

23. Li WG, Xiao JZ, Sun ZH, Kawashima S, Shah SP (2012) Interfacial transition zones in recycled aggregate concrete with different mixing approaches. Constr Build Mater 35:1045-1055

24. Xiao JZ, Li WG, Sun ZH, Lange DA, Shah SP (2013) Properties of interfacial transition zones in recycled aggregate concrete tested by nanoindentation. Cem Concr Compos 37:276-292

25. Jennings HM, Thomas JJ, Gevrenov JS, Constantinides G, Ulm F-J (2007) A multi-technique investigation of the nanoporosity of cement paste. Cem Concr Res 37(3):329-336

26. Constantinides G, Ulm F-J (2004) The effect of two types of $\mathrm{C}-\mathrm{S}-\mathrm{H}$ on the elasticity of cement-based materials: results from nanoindentation and micromechanical modeling. Cem Concr Res 34(1):67-80

27. Vandamme M, Ulm F-J, Fonollosa P (2010) Nanogranular packing of C-S-H at substochiometric conditions. Cem Concr Res 40(1):14-26

28. Němeček J, Králík V, Vondřejc J (2013) Micromechanical analysis of heterogeneous structural materials. Cem Concr Compos 36:85-92

29. da Silva WRL, Němeček J, Štemberk P (2014) Methodology for nanoindentation-assisted prediction of macroscale elastic properties of high performance cementitious composites. Cem Concr Compos 45:57-68

30. Alizadeh R, Beaudoin JJ, Raki LR (2011) Mechanical properties of calcium silicate hydrates. Mater Struct 44(1):13-28

31. Fonseca PC, Jennings HM, Andrade JE (2011) A nanoscale numerical model of calcium silicate hydrate. Mech Mater 43(8):408-419

32. Masoero E, Del Gado E, Pellenq RJ-M, Ulm F-J, Yip S (2012) Nanostructure and nanomechanics of cement: polydisperse colloidal packing. Phys Rev Lett 109(15):155503

33. Kim JH, Balogun O, Shah SP (2010) Atomic force acoustic microscopy to measure nanoscale mechanical properties of cement pastes. J Transp Res Board 2141:102-108

34. Brehm D (2012) Size diversity in cement nanoparticles optimizes packing density to give concrete its strength, Department of Civil and Environmental Engineering (CEE) in MIT. http://newsoffice.mit.edu/2012/size-diversity-incement-nanoparticles-optimizes-packing-density-to-giveconcrete-its-strength

35. Peled A, Castro J, Weiss J (2010) Atomic force microscopy examinations of mortar made by using water-filled lightweight aggregate. J Transp Res Board 2141(1):92-101 